\title{
When Further Pharmacotherapy Seems Futile
}

\author{
Joseph F. Goldberg, MD ${ }^{a, *}$
}

\begin{abstract}
I will apply, for the benefit of the sick, all measures [that] are required, avoiding those twin traps of overtreatment and therapeutic nihilism.
\end{abstract}

- Hippocratic Oath, modern version

There is an omnipresent, often tacit guiding principle within psychopharmacology to treat psychiatric disorders to the point of full remission. Given the extensive morbidity and mortality of undertreated psychiatric symptoms, alongside concerns about possible neuroprogression in many disorders,,${ }^{1-3}$ it is often considered anathema to think that any lesser outcome should ever be sufficient. ${ }^{4,5}$ Part of the taboo against "settling" for only partial improvements is as a safeguard against providing subpar care. Incomplete or poor treatment responses rightfully demand a review of diagnostic accuracy, possible missed comorbidities (including personality disorders), the appropriateness of medication choices and adequacy of trial dosages and durations, gauging patient adherence, and assessing possible drug interactions or other pharmacokinetic (eg, pharmacogenetic) factors that could lessen drug efficacy. Fewer than half of depressed patients typically receive adequate pharmacotherapy trials, ${ }^{6}$ and nonadherence to otherwise efficacious treatments can be addressed by simplifying drug regimens, targeting poor insight, managing adverse drug effects, confronting substance misuse, and strengthening treatment alliances. Suboptimal outcomes across most serious forms of mental illness are also often linked with residual symptoms, particularly in connection with psychosocial impairment, ${ }^{7}$ faster time to relapse, ${ }^{8}$ and more frequent suicide attempts. ${ }^{9}$ Finally, repeated inappropriate treatments for a wrongly diagnosed ailment are at best embarrassing and at worst disastrous.

The importance of efforts to raise awareness about the foregoing obstacles to successful treatment can never be overstated. However, even under the best of conditions with expert management, there remains an important subgroup of treatment-seeking patients with diverse psychiatric complaints whose problems and symptoms minimally improve despite numerous appropriate biological therapies. In major depression, staging models of treatment resistance project an almost exponential decay curve for the likelihood of remission with every failed adequate pharmacotherapy trial (and an accelerated rate of decline after failed electroconvulsive therapy) - such that the probability of remission after 5 failed adequate treatment trials is approximately zero. ${ }^{10}$

The literature on interventions for multidrug or multitreatment resistant patients tends mainly to urge clinicians to step up their aggressiveness when taking next steps and resist temptations to abandon hope. Stepped-up treatment aggressiveness usually means counting pills to assure adherence, redoubling efforts to address comorbid substance misuse, catching missed comorbidities, using supratherapeutically dosed medications, considering "big gun" agents such as monoamine oxidase inhibitors for refractory depression or

\footnotetext{
aDepartment of Psychiatry, Icahn School of Medicine at Mount Sinai, New York, NY

*Corresponding author: Joseph F. Goldberg, MD, 128 East Ave, Norwalk, CT 06851 (joseph.goldberg@mssm.edu).

J Clin Psychiatry 2019;80(1):18ac12276

To cite: Goldberg JF. When further pharmacotherapy seems futile. J Clin Psychiatry. 2019;80(1):18ac12276.

To share: https://doi.org/10.4088/JCP.18ac12276

(.) Copyright 2018 Physicians Postgraduate Press, Inc
}

clozapine for persistent psychosis, devising elaborate combination pharmacotherapies, and encouraging device-based interventions (eg, electroconvulsive therapy [ECT], transcranial magnetic stimulation [TMS], vagus nerve stimulation [VNS], or deep brain stimulation [DBS]). Highly treatment-refractory patients are also often referred to clinical trials, although randomized trials of novel therapies for treatment-resistant mood or psychotic disorders as listed on ClinicalTrials.gov generally do not explicitly seek to enroll patients with substantially more than 2 previous treatment failures.

Comparatively less has been written about adopting an entirely different approach designed to manage, rather than reverse or eliminate, difficult and persistent psychiatric symptoms that respond poorly to multiple appropriate interventions. In such a model, once true drug pan-refractoriness is identified, treatment orientations shift from disease modification to chronic disease management, unrealistic expectations about robust recovery are tempered, and coping becomes a primary rather than secondary focus. Trachsel and colleagues ${ }^{11}$ have suggested the term palliative psychiatry to encompass the goals of accepting the limitations of biological therapies when impairment is profound and psychiatric symptoms may be immovable. Palliative efforts aim to help patients accept and "live with" chronic symptoms as best as they can, akin to the modified goals of treatment when caring for patients with widely metastatic cancer, chronic pain, or permanent disabilities resulting from strokes or spinal cord transections.

At first glance, such an approach may seem defeatist and at variance with the concept of striving always to instill a sense of hope in patients and their families. However, it becomes disingenuous to encourage overly optimistic expectations when the chances of greater success from further iterative psychotropic drug trials are, beyond a certain point, minimal. From an evidence-based standpoint, even established or heroic pharmacotherapies for treatment-resistant depression or schizophrenia, or devices such as TMS, VNS, or ECT, have not specifically examined the effects of these interventions relative to sham procedures in highly drug-resistant disorders. Most studies operationally define treatment resistance in mood or psychotic disorders as the failure of at least 2 adequate pharmacotherapy trials, but few have examined more extreme instances of multidrug resistance (eg, > 5 failed trials, per the staging model of Petersen et $\mathrm{al}^{10}$ ). Highpotency interventions such as ECT are seldom if ever panaceas when used in highly complex, multidrug resistant cases, and success rates tend to be inversely related to episode length ${ }^{12}$ or the extent of past medication nonresponse, ${ }^{13}$ as well as the presence of certain chronic comorbidities such as borderline personality disorder. ${ }^{14}$

Insofar as a major goal of any treatment is to diminish suffering, clinicians must consider the physical and emotional burden that patients incur when they submit to multiple ineffective treatments. The very process of undergoing an inordinate number of successive medication trials with decremental probabilities of improvement could inadvertently compound psychological distress through operant conditioning. The experience of repeated unsuccessful treatment outcomes may in itself foster learned helplessness. "Maintaining hope" ought not become synonymous with the oft-quoted axiom about expecting different results when doing the same thing over and over again.

Ethical dilemmas also arise if clinicians offer desperate or heroic pharmacotherapy measures with beneficent intentions but false hope, and a lack of full transparency about the probability of success. At the same time, growth in the sheer number of available pharmacotherapies, especially antidepressants and second generation 
trials, raising the chances for an eventual spontaneous improvement that could be misattributed to the most recently introduced remedy.

Seeking formal consultation from a peer or senior colleague about "next step" treatment ideas has been a time-honored tradition for difficult cases throughout medicine. In patients with extreme multidrug resistance, this often helpful and well-intended move can be a double-edged sword. Consultants sometimes recognize a truly remediable determinant of treatment nonresponse and offer insightful suggestions; others scour a medication history in search of relevant but random medications that may not yet have been tried without commenting on the probability of success after extreme drug resistance, merely perpetuating the operant conditioning paradigm; and still others may reaffirm the accuracy of the diagnosis and appropriateness of treatment efforts to date and soberly concur with the referring clinician that all reasonable biological approaches have been tried, with little chance that further pharmacologic efforts will achieve greater gains.

There is an obviously delicate balance to be struck between openly acknowledging the low probability of benefit from additional pharmacotherapy trials beyond a certain point while at the same time redirecting patients' and families' efforts toward nonpharmacologic, non-disease-modifying interventions aimed at coping and acceptance. Care must be taken not to confuse pharmacologic futility with hopelessness about alternative ways to ease suffering or retain a sense of meaning and purpose in life. Redirected thinking toward acceptance and chronic disease management aims not to intensify feelings of hopelessness or spur suicidal thinking but, rather, to confront and manage such thoughts as cognitive distortions. Acknowledging the limits of pharmacotherapy might paradoxically improve quality of life by eliminating useless components of an extensive polypharmacy regimen, reducing cumulative side effect burden, forging more realistic expectations, strengthening coping skills, and giving permission to halt what may amount to a relentless exercise in futility.

Tenets of a "disease management approach" have been described in the context of setting realistic expectations for treatment-resistant depression. ${ }^{15}$ Such a reorientation borrows from and integrates certain core concepts of dialectical behavior therapy (DBT; such as radical acceptance and building distress tolerance skills), acceptance and commitment therapy (ACT), self-management skills training, ${ }^{16}$ and the compassionate boundary-setting used with somatically preoccupied patients in primary care. The strategy also aims to sustain a sense of hope by redirecting efforts away from repeated failures of relentless pharmacology trials and instead toward more aggressive psychotherapeutic/psychosocial treatment modalities. Other goals consistent with this model include

- providing an honest appraisal of prognosis and its modifiable versus unmodifiable determinants;

- eliminating medications of no obvious benefit that may contribute to the cumulative burden of adverse effects (particularly those that may have already imposed significant metabolic or cardiovascular hazards) or potential undesired pharmacokinetic interactions;

- helping patients differentiate acceptable from unacceptable residual symptoms or adverse drug effects;

- educating patients and families about the role of pharmacotherapy as an adjunct to psychosocial therapies, rather than the reverse;

- reorienting the goals of treatment away from disease modification and instead toward alternative outcome states, such as avoiding suicide attempts, managing chronic suicidal thinking, and avoiding emergency department visits or hospitalizations unless absolutely necessary;

- strengthening patients' capacity for resilience and ways to hone skills for coping with a chronic and possibly permanent condition;

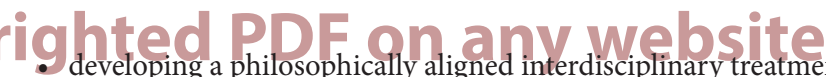

team (eg, psychotherapist, pharmacologist, case manager, primary care provider) with a convergent perspective about the goals of treatment;

- gently redirecting unrealistic expectations or magical thinking that new therapies will likely produce dramatically transformative effects and fostering their cautious rather than overzealous undertaking;

- maintaining a focus on quality of life and the pursuit of reasonable personal goals in light of, rather than as prevented by, persistent symptoms;

- recognizing the value of small gains or modest improvements (such as inroads made in insomnia, or impulsive aggression, or the ability to perform part-time volunteer work);

- helping steer patients away from potentially exploitative, costly, unnecessary evaluations that fall outside the mainstream or the pursuit of unfounded remedies with which potential hazards exceed expectable benefits (including inappropriate or nonevidence-based dosing of controlled substances);

- permitting oneself, as a clinician, the humility to accept that not all mental health disorders are biologically remediable and that even dire outcomes such as completed suicide are sometimes unpreventable despite excellent care.

Declaring pharmacologic futility is far from a casual determination and should never be confused with abandoning hope, accepting mediocrity, removing partially effective medications, rejecting sensible complementary/alternative medicine approaches, or refusing to consider future new treatments as advances in novel therapeutics further evolve. Rather, it means embracing the here-and-now reality of existing treatment limitations, fostering resilience, planning realistic goals, and breaking free from unreasonable expectations. Remission always remains the ideal outcome, but overzealous efforts to attain overidealized goals ultimately make perfection the enemy of the good. To paraphrase Winnicott, ${ }^{17}$ a "good enough" psychopharmacology often means coming to terms with the reality of imperfection. It serves no one's interests to pretend that psychiatric problems hold an immunity from that reality in ways that are somehow different from other chronic nonpsychiatric medical conditions.

\section{Published online: October 30, 2018. \\ Potential conflicts of interest: None. \\ Funding/support: None. \\ REFERENCES}

1. Kozicky JM, McGirr A, Bond DJ, et al. Bipolar Disord. 2016;18(6):511-519.

2. Salisbury DF, Kuroki N, Kasai K, et al. Arch Gen Psychiatry. 2007;64(5):521-529.

3. Moylan S, Maes M, Wray NR, et al. Mol Psychiatry. 2013;18(5):595-606.

4. Mclntyre RS, O'Donovan C. Can J Psychiatry. 2004;49(suppl 1):10S-16S.

5. Ferrier IN. J Clin Psychiatry. 1999;60(suppl 6):10-14.

6. Puyat JH, Kazanjian A, Goldner EM, et al. Can J Psychiatry. 2016;61(7):394-404.

7. Samalin L, de Chazeron I, Vieta E, et al. Bipolar Disord. 2016;18(2):164-173.

8. Judd LL, Schettler PJ, Akiskal HS, et al. Arch Gen Psychiatry. 2008;65(4):386-394.

9. Sokero TP, Melartin TK, Rytsälä HJ, et al. Br J Psychiatry. 2005;186(04):314-318

10. Petersen T, Papakostas GI, Posternak MA, et al. J Clin Psychopharmacol. 2005;25(4):336-341.

11. Trachsel M, Irwin SA, Biller-Andorno N, et al. Lancet Psychiatry. 2016;3(3):200.

12. Perugi G, Medda P, Zanello S, et al. Brain Stimul. 2012;5(1):18-24.

13. Dombrovski AY, Mulsant BH, Haskett RF, et al. J Clin Psychiatry. 2005;66(8):1043-1049.

14. Rasmussen KG. JECT. 2015;31(1):6-12.

15. Keitner GI, Ryan CE, Solomon DA. J Clin Psychiatry. 2006;67(9):1412-1421.

16. Musekamp G, Bengel J, Schuler M, et al. Patient Educ Couns. 2016;99(8):1355-1361.

17. Winnicott DW. Int J Psychoanal. 1953;34(2):89-97.

ASCP Corner offerings are not peer reviewed by the Journal but are peer reviewed by ASCP. The information contained herein represents the opinion of the author.

Visit the Society Web site at www.ascpp.org 